\title{
Nasal Reconstruction with Carved Costal Cartilage Grafts
}

\author{
${ }^{1} \mathrm{D}$ Richardson, ${ }^{2} \mathrm{RJ}$ Laycock, ${ }^{3} \mathrm{D}$ Laraway, ${ }^{4} \mathrm{AC}$ Swift
}

\begin{abstract}
Introduction: Complex reconstructive septorhinoplasty requires harvesting a large amount of tissue for grafting. Autogenous costochondral and auricular cartilage has generally considered the gold standard grafting material, if insufficient septal cartilage remains. The aim of this paper was to describe our technique and report our experience with the use of costal cartilage grafts in cases with significant nasal deformities with insufficient septal cartilage.

Design: Retrospective review of patients who underwent septorhinoplasty with costochondral grafts between 1995 and 2015.

Results: Over a 20-year period from 1995 to 2015, a total of 711 rhinoplasties were carried out at Aintree University Hospital, Liverpool. Of these, $46(5.7 \%)$ utilized costal cartilage as the material to provide skeletal support. Male to female ratio was $2: 1$, and mean age was 34 years (15-56); 22 presented with congenital deformity and 24 with acquired deformity. All had severe nasal collapse (grades III and IV).
\end{abstract}

Conclusion: Costal cartilage is the ideal material for reconstruction of severe saddle noses. There are no crossinfection risks, the donor site is low morbidity, it is easy to harvest, has a plentiful supply, and is easy to carve and sculpt. The tendency to warping is a disadvantage, but this can be prevented in most cases by careful attention to technique, and is usually easy to correct by minor revision where necessary. It is usually dimensionally stable and is able to resist infection, and where exposure occurs due to wound breakdown, it will heal without total graft loss.

Keywords: Complex reconstructive septorhinoplasty, Costal cartilage, Nasal reconstruction.

How to cite this article: Richardson D, Laycock RJ, Laraway D, Swift AC. Nasal Reconstruction with Carved Costal Cartilage Grafts. Int J Head Neck Surg 2018;9(1):7-14.

\section{Source of support: $\mathrm{Nil}$}

\section{Conflict of interest: None}

\section{INTRODUCTION}

A wide variety of surgical techniques have been described to address nasal deformity, with different etiologies and differing degrees of severity of the physical deformity.

\footnotetext{
${ }^{1-4}$ Professor

${ }^{1-4}$ Department of Oral and Maxillofacial Surgery, University Hospital Aintree, Lower Lane, Fazakerley, Liverpool, UK

Corresponding Author: Rob Laycock, Professor, Department of Oral and Maxillofacial Surgery, University Hospital Aintree, Lower Lane, Fazakerley, Liverpool, UK, e-mail: Robert.laycock@ aintree.nhs.UK
}

Many rhinoplasty techniques address "deformities" that are essentially normal morphological variations, albeit unsightly, and in these cases, where there is usually no significant deficit of normal tissues, surgery involves adjustment and repositioning of affected structures and/ or reduction of excess amount of tissue.

A small cohort of patients presenting for rhinoplasty clearly display abnormal or pathological nasal morphology, mostly characterized by severe or complete loss of the well-recognized support mechanisms for the nasal dorsum and tip, central to which is the integrity of the nasal septum. Loss of the cartilaginous septum results in severe saddle deformity and loss of nasal tip projection. Etiologies include congenital conditions (e.g., nasomaxillary hypoplasia, anterior encephalocele, orbital hypertelorism, craniofrontonasal dysplasia, facial clefts, and cleft lip and palate-associated nasal deformity) and acquired conditions (e.g., trauma, cocaine abuse, Wegener's granuloma/persistent generalized lymphadenopathy, and iatrogenic deformity). Acquired conditions may be associated with pathological change and/or loss or deficiency of the overlying soft tissue envelope, increasing the risk of wound healing problems, and occasionally necessitating soft tissue/skin replacement.

Correction of the deformity consequent upon the absence or loss of the nasal septum requires the importation of new tissue to reestablish support for the nasal dorsum and tip. A wide variety of graft materials have been used and discarded over a number of centuries, but there are still many alternatives currently in use, including alloplastic materials, xenografts, allografts (e.g., irradiated or lyophilized cartilage), and autogenous grafts of bone or cartilage. ${ }^{1}$ These different materials have their advantages and disadvantages, and the choice appears to depend to a significant extent on the individual preference of the surgeon. Alloplastic materials have the disadvantage of potential loss due to infection (early) or extrusion (late) as a result of thinning of the overlying soft tissues. Xenografts and allografts may be prone to the similar problems, but also carry a risk of cross infection, as well as resorption. Autogenous bone may be subject to resorption and gives a rigid and unnatural feel to the reconstruction, so our material of choice is autogenous cartilage, which has none of these disadvantages.

In severe deformity, where robust structural support is required, we prefer costal cartilage. This is a living, vital graft, well able to resist infection and any overlying 
wound dehiscence. There is a plentiful supply available, with a very low morbidity donor site. ${ }^{2,3}$ It is easily carved and sculpted and has good dimensional stability in most cases, and the occasional tendency to warping can be counteracted. It has the intrinsic strength and rigidity to provide secure support for the nasal dorsum and tip, but also has a natural feel and a degree of mobility that mimics to an extent the natural flexibility of the cartilaginous part of the nose.

\section{OPERATIVE TECHNIQUE}

This involves general anesthesia, with oral endotracheal intubation, supine position, and preoperative antibiotic cover.

\section{Costal Cartilage Harvest}

An incision is made above the costal margin, with dissection through subcutaneous fat to the rectus sheath. The sheath and rectus muscle are divided, to expose the underlying costal cartilages. An adequate size and shape of cartilage is identified, and harvested by dissection in the subperichondrial plane after incision of the perichondrium. Sharp dissection may be needed to separate synchondroses with the rib cartilages above and/or below. If necessary, more than one rib may be harvested, although one is usually sufficient for nasal reconstruction. Following harvest, the perichondrial incision(s) is sutured to reconstitute a perichondrial tunnel. If desired, this can be filled with diced off cuts following the carving/sculpting process in order to reconstitute the continuity of the rib. ${ }^{2}$ Rectus muscle and sheath are repaired, and skin closure with running subcuticular suture completes the closure.

\section{Cartilage Sculpting}

Where possible, sculpting of the cartilage should be done in a symmetrical fashion with regard to the rib cartilage segment to minimize the tendency for warping of the

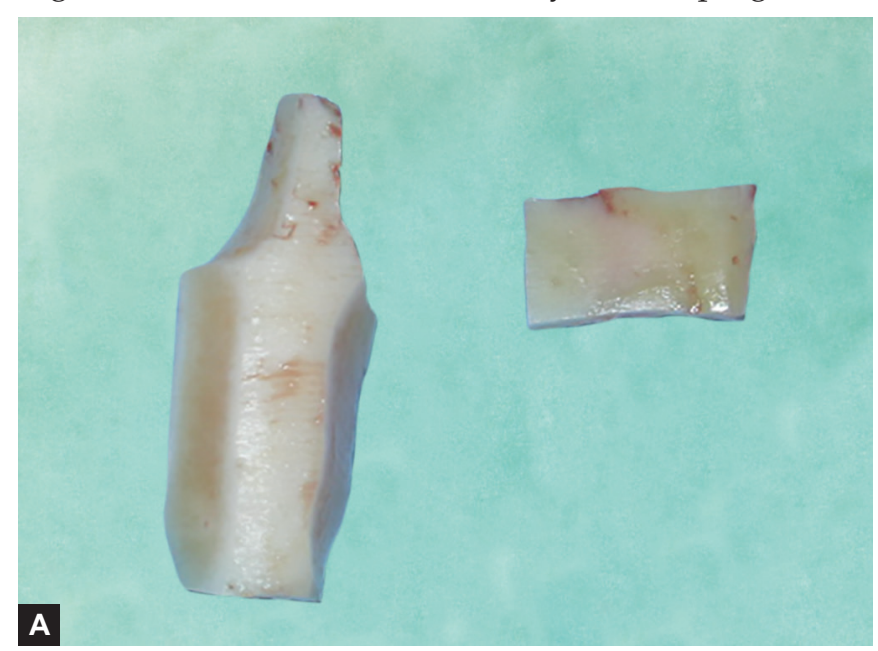

graft. The cartilage can be observed during the sculpting process, and if there is a tendency to warping, this can be counteracted by placement of mattress sutures (stainless steel wires or nonabsorbable suture), or by application of additional battens to reinforce the graft. Multiple incisions in the concave side may correct warping, if necessary with mattress suture reinforcement. Other techniques to minimize warping have been described, but have not been necessary in our series.

\section{Dorsal Augmentation}

The dorsal strut is carved symmetrically from a single costal cartilage to reproduce the desired size and shape for the graft, which should extend from radix to tip (Fig. 1). It should ensure enough width to replicate the effect of the upper lateral cartilages (which will be left unmobilized and in their original position in most cases). In severe cases, there may be a need to add further cartilage to the under surface of the dorsal strut in order to avoid a dead space between the dorsal strut and the existing bony/cartilaginous nasal dorsum.

\section{Tip Augmentation}

If the alar cartilages can be easily mobilized to a position in which good tip projection is achieved, the distal end of the dorsal strut can be narrowed to allow the alar cartilages to be sutured on either side of the graft (as well as to the columellar strut), projected so as to ensure an adequate supra tip break. In some cases, the lower lateral cartilages cannot be mobilized sufficiently to achieve tip projection, usually due to deficiency or scarring in the vestibular skin/mucosa. In this situation, the dorsal strut needs to include augmentation of the tip and be carved to reproduce the morphology of the lateral crura and tip-defining points. The tip detail can be carved from the single dorsal strut block or achieved by addition of more graft to the superior surface of the distal end of

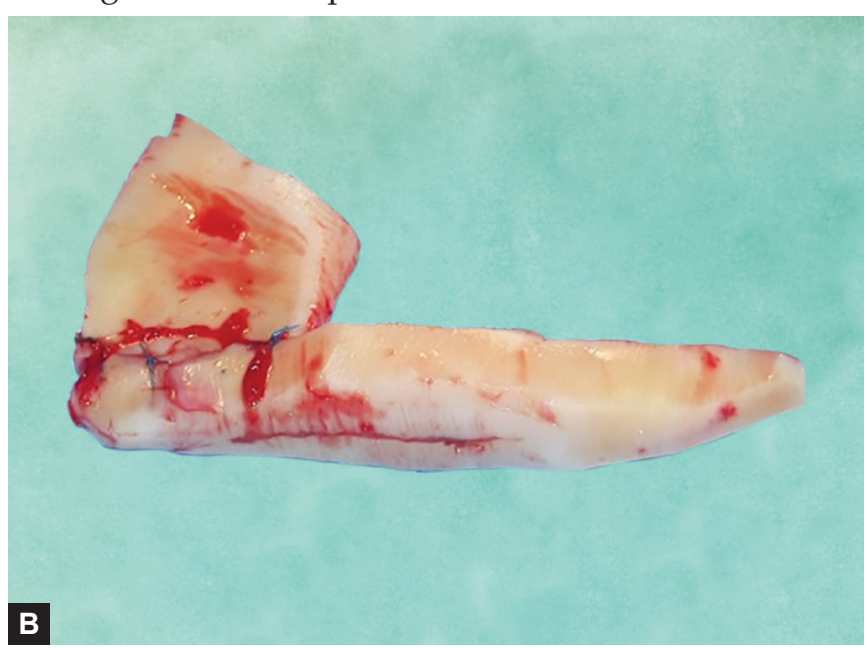

Figs 1A and B: Carved dorsal support and columella graft from rib cartilage 
the strut. In this situation, the alar cartilages are sutured to the under surface of the tip component of the dorsal strut as well as to the columellar strut (see below). There is usually sufficient elasticity in the nasal dorsal and tip skin to be able to adapt to the augmented tip cartilage graft and to achieve wound closure with an acceptable degree of tension.

\section{Columellar Strut}

This is carved symmetrically and designed to be stable when placed against the anterior nasal spine (ANS) and anterior maxilla, with a relatively wide lower third, the base of which is contoured to improve fit and lateral stability with its bony base. It should be narrower in its upper two-thirds, but of sufficient width to give the strength required to support the distal end of the dorsal strut and nasal tip (Figs 1 and 2). The columellar strut can be more prone to bending or warping because of the downward force of the newly projected tip, and care must be taken to ensure that the strut is stable in this regard. The increased thickness in this area should not result in a visible increase in columellar width, as the medial crura are usually sutured together in front of the free edge of the columellar strut, rather than on each side of it.

\section{Joining the Dorsal and Columellar Grafts}

Usually, this can only be done after insertion of both graft components, since the whole "L" shaped construct is too large to be placed in one piece. The columellar and dorsal struts can be joined by a butt joint, which requires stabilization by suturing (stainless steel wire or nonabsorbable suture), or by a mortise and tenon joint. This requires a peg-like extension (tenon) on the upper end of the columellar strut, and a hole (mortise) in the distal end of the dorsal strut-only possible if tip augmentation is carved into the single block dorsal strut. Both the tenon

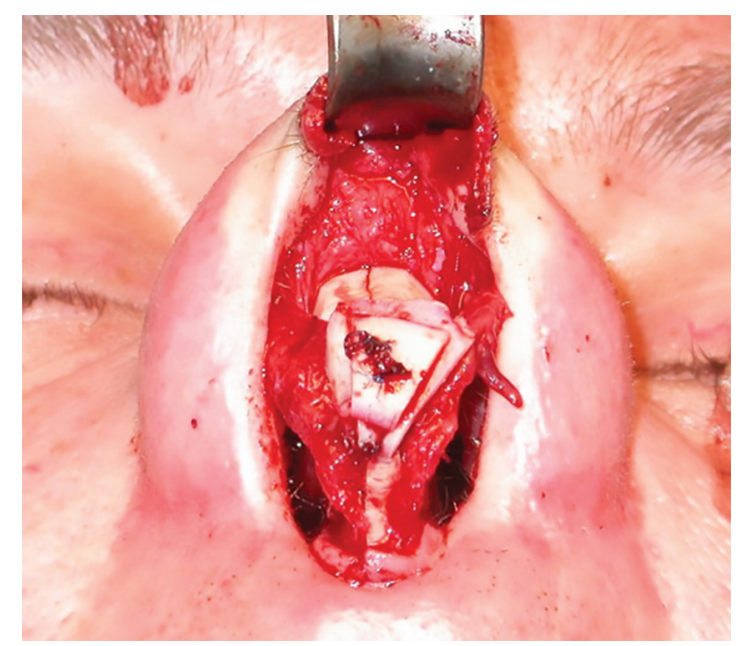

Fig. 2: Open rhinoplasty approach: carved costal cartilage grafts and tip graft displayed and the mortise are easily created using dermatology punches - that for the tenon being one size smaller the mortise. Once in position, the joint is stable and does not require additional fixation.

\section{Insertion of the Graft}

This requires an open rhinoplasty approach, with exposure of the whole nasal dorsum, and separation of the lower lateral (alar) cartilages and membranous septum with exposure of the caudal edge of the residual septum (if present). Dissection continues between the medial crura to the ANS and adjacent surface of the maxilla to create a pocket for the columellar strut. If access is required to contour the ANS/pyriform sill or to fix the base of the columellar strut to ensure stability, an upper sublabial intraoral incision can provide this.

The dorsal and columellar struts are placed individually, and the skin redraped; further sculpting/ adjustments may be carried out to ensure the optimum esthetic result possible is achieved without significantly compromising wound closure with excess tension. Once this has been completed, the joint between the columellar and dorsal struts is established, the posterior edge of the columellar strut may be sutured to the caudal edge of the nasal septum (if present), and the alar cartilages (medial crura) are sutured to the anterior margin of the columellar strut, and if needed, interdomal sutures may be placed to reconstitute the tip anatomy.

\section{Closure}

The open rhinoplasty incision is closed with sutures.

Temporary fixation of the dorsal strut to avoid early displacement can be achieved by transfixion with one or two straight suture needles left projecting through the dorsal nasal skin. Nasal tapes and splints can be applied around the projecting ends of the needles, and all are left in situ for 10 days prior to removal at outpatient follow-up.

\section{RESULTS}

Over a 20-year period from 1995 to 2015, a total of 711 rhinoplasties were carried out at Aintree University Hospital, Liverpool. Of these, 46 (5.7\%) utilized costal cartilage as the material to provide skeletal support. Male to female ratio was 2:1, and mean age was 34 years (15-56); 22 presented with congenital deformity and 24 with acquired deformity. All had severe nasal collapse (grades III and IV).

The cohort consists of patients with diverse etiologies, including craniofacial syndromes (16 patients), cleft lip and palate (5 patients), trauma (7 patients), cocaine abuse (6 patients), trauma and cocaine (1 patient), granulomatosis with polyangiitis (5 patients), postradiotherapy (3 patients), 
and three patients with other conditions (meningococcal septicemia, iatrogenic, and AAA syndrome).

Other operations planned in conjunction with the nasal reconstruction included forehead augmentation, orbital osteotomy for hypertelorism, and facial bipartition in patients with craniosynostosis syndromes, and one facial cleft repair. Le Fort 1 maxillary osteotomy (2 patients-1 cleft lip and palate, 1 orthognathic).

Postoperative complications (with 4 weeks of surgery) were seen in four patients $(10 \%)$ and included wound breakdown ${ }^{1}$ and infection. ${ }^{3}$ These all occurred in patients with a pathological etiology for the nasal deformity, with compromised soft tissue cover. Late complications were seen in five patients (12\%) and included cartilage warping (two patients-both corrected by subsequent minor revision surgery) and soft tissue contraction (two patients-both occurring in patients who had received radiotherapy for malignant nasal tumors) and skin breakdown following thinning of forehead flaps as a secondary procedure (one patient).

Examples of the esthetic results are shown in Figures 3 to 6. Only three patients expressed dissatisfaction with
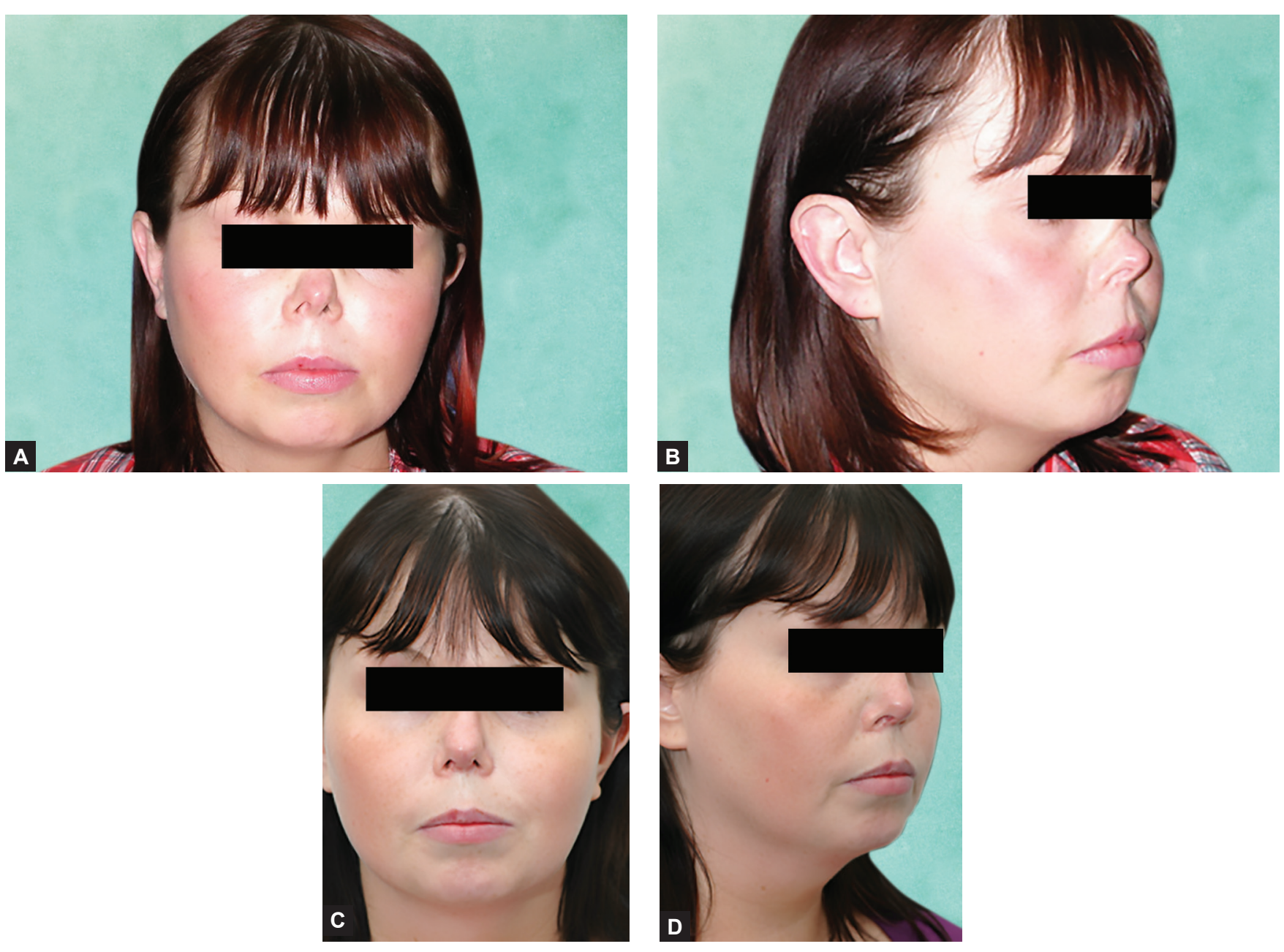

Figs 3A to D: (A and B) Saddle deformity due to granulomatosis with polyangiitis; (C and D) Post reconstruction with carved costal cartilage graft

the final outcome, all related to deficiencies in the soft tissues rather than problems related to the costal cartilage.

There were no significant costal cartilage donor site complications.

\section{DISCUSSION}

Autogenous cartilage is accepted by most authors as the gold standard for nasal reconstruction. ${ }^{2-4}$ However, many of these go on to describe their experience with a variety of other materials, mostly allografts or xenografts, and choose to use other materials for a variety of reasons related to the perceived problems or difficulties associated with use of autogenous cartilage.

Donor sites for autogenous cartilage include nasal septum, ear, and chest. Where small amounts of cartilage are required for contour augmentation where nasal support is not significantly compromised, septum and auricular cartilage are often sufficient, and these materials can be used to augment support, particularly in the columella. However, for more severe deformities, with significant loss of dorsal and tip support, often with 

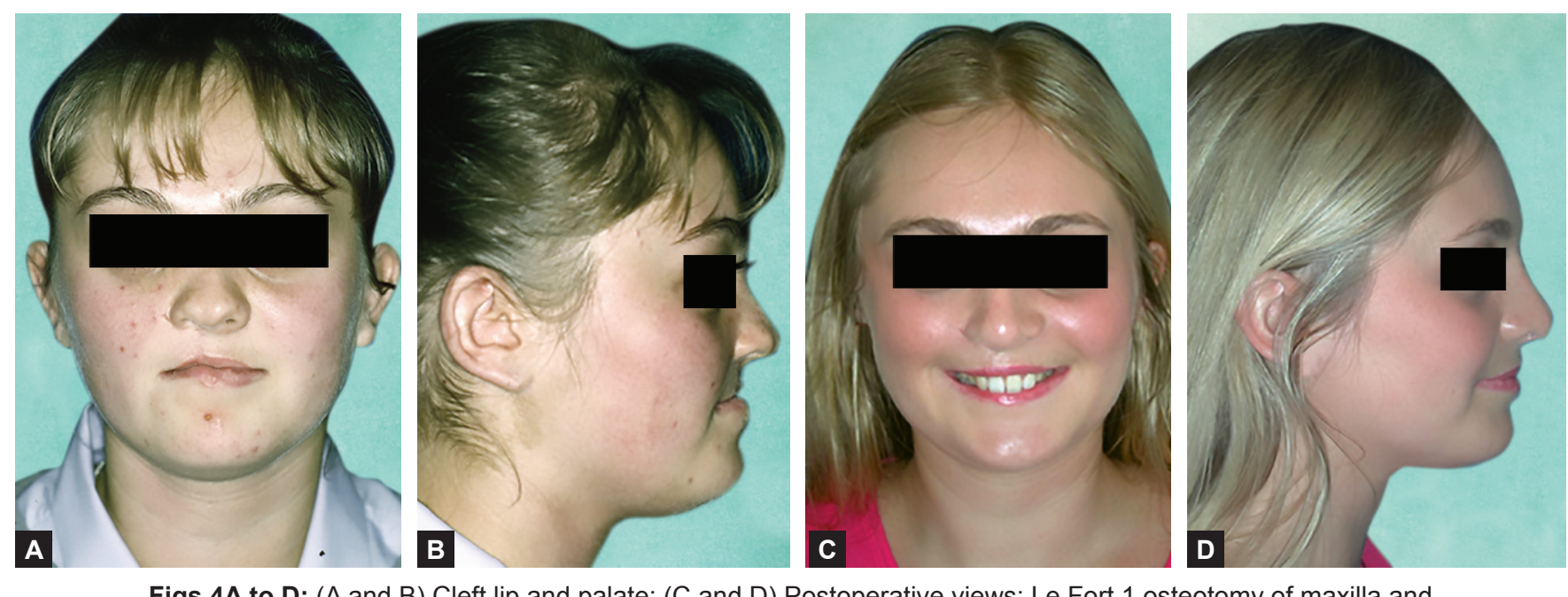

Figs 4A to D: (A and B) Cleft lip and palate; (C and D) Postoperative views: Le Fort 1 osteotomy of maxilla and costal cartilage dorsal support and columella strut
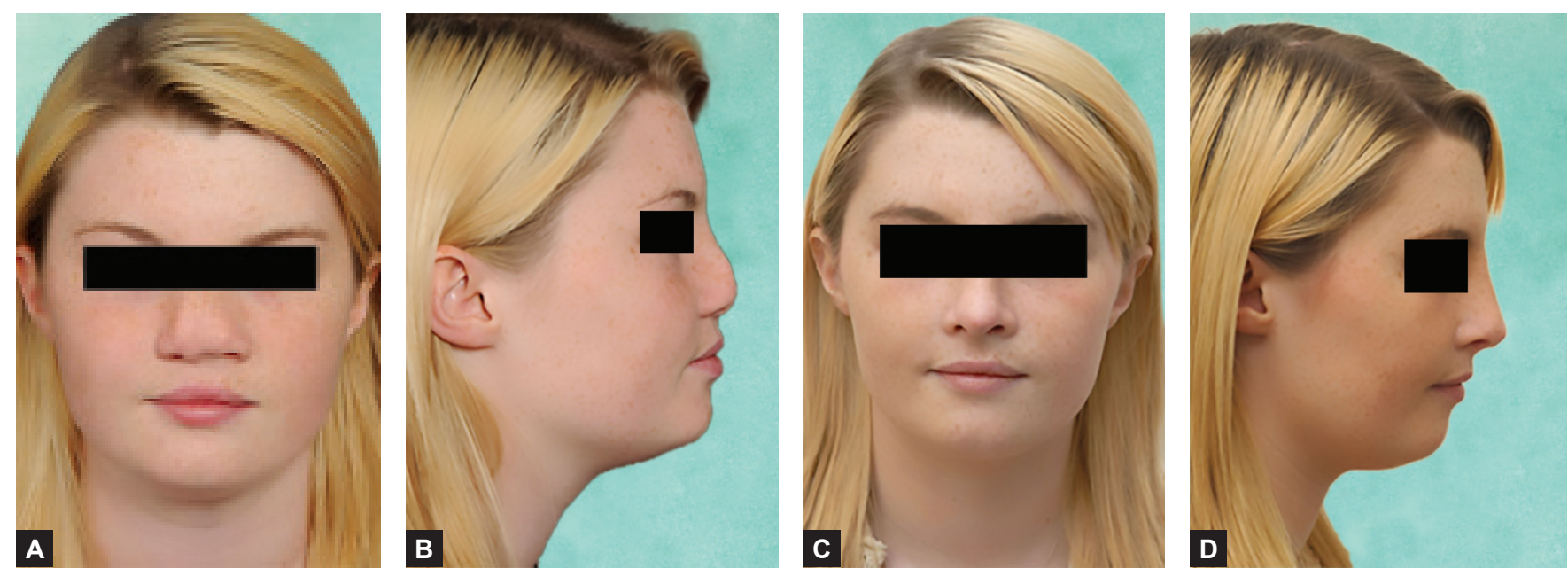

Figs 5A to D: (A and B) Facial cleft and hypertelorism; (C and D) Postoperative views. Facial cleft and Hypertelorism: medial orbital wall osteotomies, costal cartilage dorsal support and columella strut
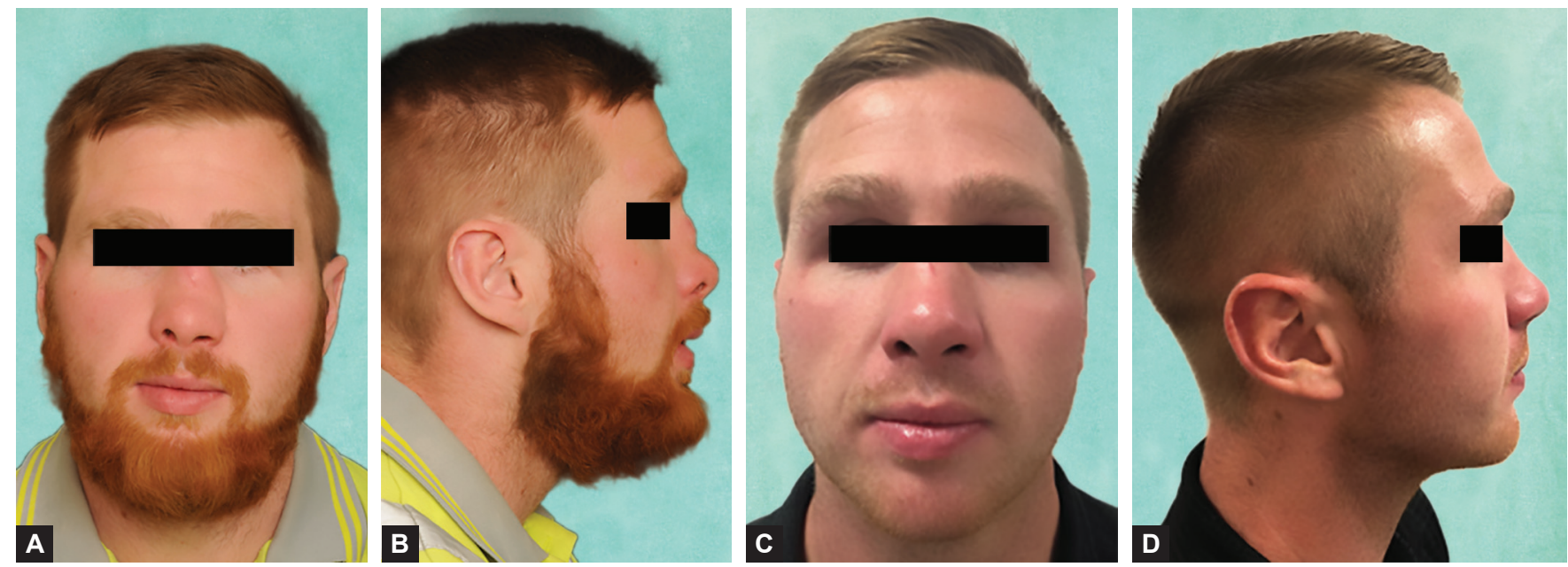

Figs 6A to D: (A and B) Post traumatic saddle deformity; ( $C$ and $D)$ Postoperative nasal reconstruction with costal cartilage

deficiencies both of the septum and in the overlying soft tissue envelope, material with greater strength may be required to provide stable structural support. Costal cartilage is able to provide the strength and support required, is available in plentiful supply, and is easy to carve and sculpt to recreate the desired surface anatomy 
of the nose, and an acceptable esthetic outcome. Cited difficulties in using costal cartilage include donor site morbidity, tendency to warp, and resorption. In our opinion, these potential disadvantages are outweighed by the advantages of using autogenous material, which is a living graft and able to resist and survive postoperative exposure due to wound dehiscence and infection, and which is well tolerated in the long term.

\section{Donor Site}

Costal cartilage donor site is associated with a low morbidity. ${ }^{2,3}$ Thoracic deformity has been described in young patients with significant numbers of costal cartilage harvested, usually for microtia reconstruction. Nasal reconstruction in this context is usually undertaken in older patients, and one costal cartilage is often sufficient for the reconstruction. Subperichondrial harvest, preserving and repairing the perichondrial tunnel, and if needed reconstituting the rib using diced off cuts after the cartilage carving is complete have all been shown to yield excellent results at the donor site, and this is mirrored in our experience. Use of costochondral graft incorporating bone, costochondral junction, and costal cartilage has been advocated (see below). There is a higher risk of pleural damage compared with cartilage only, since it is necessary to harvest a higher rib where the pleura is directly deep to the perichondrium and periosteum on the deep surface of the rib, compared with a lower rib (C6 and below) where the abdominal wall muscles provide a "safety layer" deep to the perichondrium. Despite this, the risk of pneumothorax is extremely low. In the series reported by Chummun et al. of 150 costochondral graft, pleural damage was reported in one case. In the metaanalysis by Wee et al. ${ }^{5}$ pneumothorax risk is quoted at $0 \%$, and hypertrophic scar at $5.45 \%$. The low incidence of donor site complications is mirrored in our series.

\section{Costochondral Graft}

Use of a costochondral graft, with bone for the upper third of the nose, and cartilage for the lower two-thirds, has been described ${ }^{4,6-8}$ often, but not always, in children with congenital deformity. In skeletally mature patients, there is little or no advantage to incorporating bone and costochondral junction, with the disadvantage of potential bone remodeling and/or resorption. The rationale for costochondral graft in skeletally immature patients is the incorporation of the costochondral growth plate for nasal lengthening during subsequent facial growth. Our policy is to wait for skeletal maturity (or close to it) to avoid the uncertainties of the effects of future growth. Growth of costochondral grafts has been shown to be unpredictable in the context of facial growth after temporomandibular joint reconstruction, and overgrowth has been described in the context of rhinoplasty. ${ }^{4,6,9}$ While some studies report a degree of costochondral graft growth, there is no good evidence in the literature to support the concept of "interceptive" rhinoplasty during growth, or the efficacy of costochondral grafts to mimic normal nasal growth. In the largest series ${ }^{4}$ involving 150 grafts in 107 patients, 42 patients required subsequent repeat costochondral grafting or significant revision of the graft.

\section{Warping}

This is a significant phenomenon and can occur within minutes of carving, or develop over months or years. Intraoperative maneuvers can reduce or eliminate the early warping and are outlined above. Where possible, sculpting of the cartilage should be done in a symmetrical fashion with regard to the rib cartilage segment to minimize the tendency for warping of the graft. ${ }^{10,11}$ The cartilage can be observed during the sculpting process, ${ }^{12}$ and if there is a tendency to warping, this can be counteracted by placement of mattress sutures (stainless steel wires or nonabsorbable suture), or by application of additional battens to reinforce the graft. A counterbalancing technique designed to make the warping forces equal and opposite has been described. ${ }^{13}$ Multiple incisions in the concave side may correct warping, if necessary with mattress suture reinforcement, and similar incisions on both sides ("Accordion technique") ${ }^{14}$ may prevent warping. Use of a $\mathrm{K}$ wire as an internal cartilaginous splint has also been described ${ }^{15}$ and avoidance of carving by using an "edge on" technique has been demonstrated. ${ }^{16}$

Warping is reported in 0 to $38.8 \%,{ }^{12,14,16-19}$ with Wee reporting an incidence of $3.8 \%$ in a meta-analysis involving 458 patients. In our series, there were only two cases of warping $(4.9 \%)$ requiring revision, both involving deviation of the dorsum. This can be addressed by an "I to V" incision at the depth of the concavity, straightening of the dorsal graft opening up the linear cartilaginous incision to a " $\mathrm{V}$ " configuration without the need to remove the graft. The "V" can be filled with a small graft to maintain the opening and must be stabilized with suture or wire to avoid subsequent displacement. More significant deviation may require removal and remodeling of the costal cartilage, but this has not occurred in our series.

\section{Resorption}

Volume stability is an important facet of any nasal graft, and alloplastic materials have the advantage of no significant change in dimensions following insertion. Homologous cartilage although not a viable material is 
prone to resorption, ${ }^{12}$ and viable living grafts of bone or cartilage are not immune from this process. Normal bone is subject to remodeling and resorption, even in the absence of complications, such as infection or soft tissue breakdown, and in nonstress bearing situations, particularly onlay grafting, the lack of functional stimulation may exacerbate this tendency. Membranous bone (e.g., calvarium) is less prone to resorption than cartilaginous bone (e.g., rib) and rigid fixation may help to reduce resorption.

Costal cartilage, once established, is a living graft with very little tendency for resorption in the absence of infection or soft tissue dehiscence, and reported rates of volume loss vary from 3 to $6 \% .^{17-19}$ However, the meta-analysis by Wee reports an incidence of $0.22 \%$ in 485 patients. In the absence of infection or soft tissue problems, there were no cases of resorption in our series. Where breakdown occurred, this happened in cases where there was pathological abnormality in the soft tissue envelope (GPA, cocaine use, radiotherapy). However, even in these situations, conservative management of cartilage exposure with topical silver sulfadiazine cream (Flamazine $^{\mathrm{TM}}$ ) resulted in successful would healing, albeit with locaized resorption of the underlying cartilage. The defect, once healed, is amenable to secondary reconstruction, without loss of the whole graft. This contrasts with most other materials, where exposure results in total graft loss.

\section{Infection}

Incidence of infection was reported at $0.56 \%$ by Wee et al,5 although a range of incidences $(0-8.4 \%)$ are reported in individual series. ${ }^{17-29}$ In our series, there was only one case of postoperative infection with Staph. aureus infection successfully treated with antibiotics and aspiration. There was no subsequent resorption and the graft remained stable and functional over a 20-year follow-up.

\section{Revision Surgery}

Despite the low rate of surgical complications associated with use of costal cartilage for nasal reconstruction, Wee et al. ${ }^{5}$ report a revision surgery rate of $14 \%$. The reasons for this are not stated, but the revision rate is probably related to the difficulties in achieving the desired esthetic outcome in rhinoplasty surgery generally, and a revision rate is accepted in any nasal esthetic procedures. In severe deformity requiring costal cartilage grafting, the requirement for revision procedures is perhaps unsurprising, irrespective of the reconstructive material used.

\section{CONCLUSION}

Costal cartilage is the ideal material for reconstruction of severe saddle noses. There are no cross-infection risks, the donor site is low morbidity, it is easy to harvest, has a plentiful supply, and is easy to carve and sculpt. The tendency to warping is a disadvantage, but this can be prevented in most cases by careful attention to technique, and is usually easy to correct by minor revision where necessary. It is usually dimensionally stable and is able to resist infection, and where exposure occurs due to wound breakdown, it will heal without total graft loss.

\section{REFERENCES}

1. Dresner HS1, Hilger PA. An overview of nasal dorsal augmentation. Semin Plast Surg. 2008 May;22(2):65-73.

2. Kawanabe $Y, N a g a t a S$. Anew method of costal cartilage harvest for total auricular reconstruction: part I. Avoidance and prevention of intraoperative and postoperative complications and problems. Plast Reconstr Surg. 2006 May;117(6): 2011-2018.

3. Varadharajan K1, Sethukumar P1, Anwar M1, Patel K1. Complications Associated With the Use of Autologous Costal Cartilage in Rhinoplasty: A Systematic Review. Aesthet Surg J. 2015 Aug;35(6):644-652.

4. Chummun S, McLean NR, Anderson PJ, David DJ. Along-term evaluation of 150 costochondral nasal grafts. J Plast Reconstr Aesthetic Surg. 2013;66:1477-1481

5. Wee JH, Park M-H, Oh S, Jin H-R. Complications Associated With Autologous Rib Cartilage Use in Rhinoplasty A Metaanalysis. JAMA Facial Plast Surg. 2015;17(1):49-55.

6. Holmes AD, Lee SJ, Greensmith A, Heggie A, Meara JG. Nasal Reconstruction for Maxillonasal Dysplasia. J Craniofac Surg 2010 Mar; 21(2): 543-551.

7. Gurley JM, Pilgram T, Perlyn CA, Marsh JL. Long-term outcome of autogenous rib graft nasal reconstruction. Plast Reconstr Surg. 2001 Dec;108 (7):1895-905.

8. Li D, Mao H, Yu Z, Xu L, Yuan J, Wei M. Primary aesthetic correction of nasal anomaly with costal graft in treating orbital hypertelorism. J Cranio Max Fac Surg. 2018;46::6-10

9. Baek RM, Lee Y, Song YT. Overgrowth of a costochondral graft in nasal reconstruction. J Craniofac Surg. 2005 Jul;16(4):736-740.

10. Gibson T, Davies WB. The distortion of autogenous cartilage grafts: its causes and prevention. Br J Plast Surg. 1958;010:257-274.

11. Kim DW, Shah AR, Toriumi DM. Concentric and eccentric carved costal cartilage: a comparison of warping. Arch Facial Plast Surg. 2006;8:42-46

12. Balaji SM. Costal cartilage nasal augmentation rhinoplasty: Study on warping. Ann Maxillofac Surg. 2013 Jan-June;3(1): 20-24.

13. Kapil S. Agrawal, Manoj Bachhav, and Raghav Shrotriya. Namaste (counterbalancing) technique: Overcoming warping in costal cartilage. Indian J Plast Surg. 2015 May-Aug;48(2):123-128.

14. Ozturano, Aksoy F, Veyseller B, Apuhan T, Yildirim YS. Severe saddle nose: choices for augmentation and application of accordion technique against warping. Aesthetic Plast Surg. 2013 Feb;37(1):106-116.

15. Gunter JP, Clark CP, Friedman RM. Internal stabilization of autogenous rib cartilage grafts in rhinoplasty: a barrier to cartilage warping. Plast Reconstr Surg. 1997;100: 161-169. 
16. Al-Qattan MM. Augmentation of the nasal dorsum with autogenous costal cartilage using the "edge-on" technique. Ann Plast Surg. 2007 Dec;59(6):642-644.

17. Yeon Hee Joo, Yong Ju Jang. Comparison of the Surgical outcomes of Dorsal Augmentation using Expanded Polytetrafluoroethylene or Autologous Costal Cartilage. JAMA Facial Plast Surg. 2016;18(5);327-332.
18. Wee JH, Mun SJ, Na WS, Kim H, Park JH, Kim DK, Jin HR. Autologous vs Irradiated Homologous Costal Cartilage as Graft Material in Rhinoplasty183-188. JAMA Facial Plast Surg 2017 May 1;19(3):183-186.

19. Moon BJ, Lee HJ, Jang YJ. Outcomes Following Rhinplasty using Autologous Costal Cartilage. Arch facial Plast Surg. 2012;14(3):175-180. 